\title{
AUTOMORPHISMS OF A FREE ASSOCIATIVE ALGEBRA OF RANK 2. I
}

\author{
BY \\ ANASTASIA J. CZERNIAKIEWICZ
}

\begin{abstract}
Let $R_{\langle 2\rangle}=R\langle x, y\rangle$ be the free associative algebra of rank 2, on the free generators $x$ and $y$, over $R$ ( $R$ a field, a Euclidean domain, etc.). We prove that if $\varphi$ is an automorphism of $R_{\langle 2\rangle}$ that keeps $(x y-y x)$ fixed (up to multiplication by an element of $R$ ), then $\varphi$ is tame, i.e. it is a product of elementary automorphisms of $R_{\langle 2\rangle}$. This follows from a more general result about endomorphisms of $R_{\langle 2\rangle}$ via a theorem due to $\mathrm{H}$. Jung [6] concerning automorphisms of a commutative and associative algebra of rank 2 .
\end{abstract}

Introduction. Let $R$ be a commutative domain with 1. We will further assume that every invertible matrix with coefficients in $R$ is a product of elementary matrices (e.g. $R$ a field, $R$ a Euclidean domain). Let $R_{\langle n\rangle}$ be the free associative algebra of rank $n$ over $R$.

It has been conjectured (see P. M. Cohn [1, p. 33]) that every $R$ automorphism of $R_{\langle n\rangle}$ is tame (i.e. a product of elementary automorphisms of $R_{\langle n\rangle}$ ).

The conjecture has been proved true for a free Lie algebra by P. M. Cohn [3], and for $R(\tilde{x}, \tilde{y})$, the free commutative and associative algebra of rank 2 (i.e. the polynomial ring in 2 commuting indeterminates $\tilde{x}$ and $\tilde{y}$ ) by Jung [6].

We shall prove here that a certain class of automorphisms of $R_{\langle 2\rangle}$ satisfies the conjecture, namely that if $R\langle x, y\rangle$ is the free associative algebra over $R$, in the free generators $x$ and $y$, any automorphism $\varphi$ that keeps $(x y-y x)$ fixed (up to a scalar multiple) is tame.

We also give a partial answer to the following question (see [4, p. 197]): If $A$ and $B$ are two elements of $R_{\langle 2\rangle}$ so that $[A, B]=\lambda[x, y], \lambda$ a unit of $R$, is it true that $A$ and $B$ generate $R_{\langle 2\rangle}$ ? We prove that the answer is "yes" if $A=x+P, B=y+Q$, where $P$ and $Q$ satisfy condition (iii) below.

The following is an outline of our proof.

Given $\varphi \in \mathrm{Aut}_{R}(R\langle x, y\rangle)$ so that $\varphi$ keeps $(x y-y x)$ fixed, Jung's theorem allows us to reduce the problem of proving that $\varphi$ is tame to the case in which the canonical projection of $\varphi$ to $R(\tilde{x}, \tilde{y})$ is the identity. More generally we prove

Received by the editors October 27, 1970.

AMS 1970 subject classifications. Primary 16A06, 16A72; Secondary 20F55, 16A02.

Key words and phrases. Free associative algebra, endomorphisms, automorphisms, elementary automorphisms, tame automorphisms, wild automorphisms, polynomial rings.

Copyright (C) 1971, American Mathematical Society 
THEOREM. If $\varphi$ is an R-endomorphism of $R\langle x, y\rangle$ so that

(i) $\varphi(x)=x+P(x, y), \varphi(y)=y+Q(x, y)$,

(ii) $[x+P, y+Q]=[x, y]$, and

(iii) $P$ and $Q$ are free of terms of zero and first degree and they do not contain terms which are pure in " $y$ ", then $\varphi$ is an elementary automorphism of $R_{\langle 2\rangle}$.

This is proved using a result of P. M. Cohn about centralizers of homogeneous polynomials of $R_{\langle 2\rangle}$ (see Lemma 1) and a purely combinatorial argument which shows that the system of equations $[P, y]+[x, Q]+[P, Q]=0$, with condition (iii) for $P$ and $Q$, has as the only solution $P=0, Q=f(x)$.

It is still an open question whether any automorphism of $R_{\langle 2\rangle}$ will or will not keep $(x y-y x)$ fixed.

I want to express my gratitude to Professor Wilhelm Magnus, my thesis adviser, whose suggestions and constant encouragement made this work possible. Let me also express my thanks to the referee of this paper; in particular the notation used here owes much to him.

NoTE. After this paper was written we have succeeded in proving that every automorphism of $R\langle x, y\rangle$ is indeed tame. In fact we have proved that the map Aut $(R\langle x, y\rangle) \rightarrow \operatorname{Aut}(R(\tilde{x}, \tilde{y}))$ induced by the abelianization functor is a monomorphism, and this together with Jung's result for the polynomial algebra gives the assertion for $R\langle x, y\rangle$. The proof uses the techniques developed in the present paper. See Automorphisms of a free associative algebra of rank 2. II.

Definitions and notation. $R\langle x, y\rangle=R_{\langle 2\rangle}$ denotes the free associative algebra of rank 2, over $R$, in the free generators $x$ and $y$ (i.e. the polynomials with coefficients in $R$, in the noncommuting indeterminates $x$ and $y$ ). Every element of $R_{\langle 2\rangle}$ will be called a polynomial.

Let $P$ be a polynomial, then $P$ can be written uniquely as

$$
P=P_{0}+P_{1}+\cdots+P_{r}
$$

where $P_{i}, i=0,1, \ldots, r$, is a homogeneous polynomial of degree $i$. We write $d(P)$ $=$ degree of $P=r$, provided $P_{r} \neq 0 . P$ can also be written uniquely as

$$
P=P^{(0)}+P^{(1)}+\cdots+P^{(s)},
$$

where $P^{(j)}, j=0,1, \ldots, s$, is a polynomial that has constant degree in the variable $x$ and that degree is equal to $j$. As a rule we will suppress the parentheses of an upper index whenever there is no danger of confusion with an exponent.

If $\varphi$ is an $R$-endomorphism of $R_{\langle 2\rangle}$ we assume $\varphi(1)=1$, which implies that $\varphi$ restricted to $R \cdot 1 \subset R_{\langle 2\rangle}$ is the identity of $R$. If $\varphi \in \operatorname{End}_{R}(R\langle x, y\rangle)$ then $\varphi$ is completely characterized by its values in $x$ and $y$, i.e. $\varphi(x)$ and $\varphi(y)$ determine $\varphi$. 
The elementary automorphisms of $R\langle x, y\rangle$ are the following automorphisms:

(i) $\sigma \in \mathrm{Aut}_{R}(R\langle x, y\rangle), \sigma(x)=y, \sigma(y)=x$,

(ii) $\varphi_{\alpha, \beta} \in \operatorname{Aut}_{R}(R\langle x, y\rangle), \alpha, \beta$ units of $R, \varphi_{\alpha, \beta}(x)=\alpha x, \varphi_{\alpha, \beta}(y)=\beta y$,

(iii) $\tau_{f(y)} \in \operatorname{Aut}_{R}(R\langle x, y\rangle)$, where $f(y)$ is any polynomial that does not depend on $x, \tau_{f(y)}(x)=x+f(y), \tau_{f(y)}(y)=y$.

The same definitions characterize the elementary automorphisms of $R(\tilde{x}, \tilde{y})$, the free commutative and associative algebra of rank 2 (i.e. the polynomial algebra over $R$, in some commuting independent variables $\tilde{x}$ and $\tilde{y}$ ).

An automorphism is called tame if it is a product of elementary automorphisms.

LEMMA 1. Let $M$ and $N$ be two nonzero homogeneous polynomials of $R_{\langle 2\rangle}$ so that $M N=N M$. Then there exist a nonzero polynomial $K$ of $R_{\langle 2\rangle}, r, s$ nonnegative integers and nonzero $\alpha, \beta, \lambda, \mu \in R$ so that the following is satisfied:

$$
\alpha M=\lambda K^{r}, \quad \beta N=\mu K^{s} .
$$

Proof. If $d(M)=d(N)=0$ then $M$ and $N$ reduce to constants and the result follows by taking $K=1$.

Let us assume now, as an inductive hypothesis, that the result holds whenever $d(N) \leqq d(M)<m$ and let us prove it for the case $d(N) \leqq d(M)=m>0$.

By the theorem of P. M. Cohn [2, p. 351] that states that the homogeneous polynomials of $F\langle x, y\rangle$ ( $F$ the field of quotients of $R$ ) form a free multiplicative semigroup, we know that (up to multiplication by constants) we can write $M$ and $N$ as a product of irreducible factors and that decomposition is unique, order included.

It follows then from

$$
M N=N M
$$

that there is a polynomial $H, d(H) \leqq d(M), a \in R, a \neq 0$ so that

$$
a M=N H \text {. }
$$

In case $d(M)=d(N)$, we have $d(H)=0$ and the result follows by taking $K=M$.

In case $d(M)>d(N)>0$, we have from (1) and (2) that $N H N=N N H$ as $N \neq 0$ and $R_{\langle 2\rangle}$ is a domain the following holds: $H N=N H$ where $d(N)<d(M), d(H)$ $<d(M)=m$.

By inductive hypothesis there follows the existence of a nonzero polynomial $K$, $r, s$ positive integers and nonzero $\alpha, \beta, \lambda, \mu \in R$ so that $\alpha N=\lambda K^{r}, \beta H=\mu K^{s}$. And from (1) it follows that $\alpha^{\prime} M=\mu^{\prime} K^{r+s}, \alpha^{\prime}, \mu^{\prime}$ nonzero.

Finally in the case $d(M)>d(N)=0, N$ reduces to a constant and we may take $K=M$. This completes the proof.

Corollary. Let $P$ be a polynomial of $R\langle x, y\rangle$ so that $P \cdot x=x \cdot P$. Then $P=f(x)$.

The proof follows by applying Lemma 1 to the homogeneous components of each term. 
REMARKS. 1. Let $\varphi$ be an endomorphism of $R_{\langle 2\rangle}$ defined as follows:

$$
\varphi(x)=a x+b y, \quad \varphi(y)=c x+d y, \quad a, b, c, d \in R,
$$

where $(a d-b c)$ is a unit of $R$. Then $\varphi$ defines an automorphism of $R_{\langle 2\rangle}$ and moreover $\varphi$ is tame. In fact: we have assumed that every invertible matrix with coefficients in $R$ is a product of elementary matrices.

2. Let $\varphi$ be an endomorphism of $R_{\langle 2\rangle}$ so that

$$
\varphi(x)=a+P(x, y), \quad \varphi(y)=b+Q(x, y), \quad a, b \in R,
$$

where $P$ and $Q$ are polynomials of $R_{\langle 2\rangle}$ that do not contain terms of zero degree. Let $\psi$ be the tame automorphism of $R_{\langle 2\rangle}$ defined as follows:

$$
\psi(x)=x-a, \quad \psi(y)=y-b .
$$

Then $\varphi^{\prime}=\varphi \circ \psi$ satisfies

$$
\varphi^{\prime}(x)=P(x, y), \quad \varphi^{\prime}(y)=Q(x, y) ;
$$

i.e. we can assume that, modulo a tame automorphism, every endomorphism of $R_{\langle 2\rangle}$ sends $x$ and $y$ into polynomials without terms of zero degree.

LEMma 2. Let $\varphi$ be an automorphism of $R_{\langle 2\rangle}$ so that

$$
\begin{aligned}
& \varphi(x)=a x+b y+P(x, y), \quad a, b, c, d \in R, \\
& \varphi(y)=c x+d y+Q(x, y),
\end{aligned}
$$

and $P$ and $Q$ are polynomials of $R_{\langle 2\rangle}$ which are free of terms of zero and first degree. Let $\varphi_{L}(=$ linear part of $\varphi) \in$ End $\left(R_{\langle 2\rangle}\right)$ be defined as

$$
\varphi_{L}(x)=a x+b y, \quad \varphi_{L}(y)=c x+d y .
$$

Then $\varphi_{L}$ is an automorphism of $R_{\langle 2\rangle}$. Moreover $\left(\varphi_{L}\right)^{-1}=\left(\varphi^{-1}\right)_{L}\left(=\right.$ linear part of $\left.\varphi^{-1}\right)$.

Proof. It suffices to observe that $\varphi^{-1}$ is given by

$$
\begin{aligned}
& u=\varphi^{-1}(x)=a^{\prime} x+b^{\prime} y+P^{\prime}(x, y), \\
& v=\varphi^{-1}(y)=c^{\prime} x+d^{\prime} y+Q^{\prime}(x, y), \\
& a^{\prime}, b^{\prime}, c^{\prime}, d^{\prime} \in R,
\end{aligned}
$$

where $P^{\prime}$ and $Q^{\prime}$ are some polynomials of $R_{\langle 2\rangle}$ which are free of terms of zero and first degree. Notice also that if $M$ is a monomial of degree $\geqq 2$, then all the terms of $M(u, v)$ are of degree $\geqq 2$. It follows then from $\varphi^{-1} \circ \varphi(x)=x, \varphi^{-1} \circ \varphi(y)=y$, that the matrix $\left(\begin{array}{ll}a^{\prime} & b^{\prime} \\ c^{\prime} & a^{\prime}\end{array}\right)$ is the inverse of the matrix $\left(\begin{array}{ll}a & b \\ c & d\end{array}\right)$ which proves the assertion.

These lemmas and remarks show that every $\varphi \in \operatorname{Aut}_{R}(R\langle x, y\rangle)$ after combining with a tame automorphism takes the form $\varphi(x)=x+P(x, y), \varphi(y)=y+Q(x, y)$, where the degree of the lowest nontrivial terms of $P$ and $Q$ is at least 2 .

Lemma 3. Let $c$ and $d$ be positive integers which are relatively prime, $c \geqq d$; let $r, s$ be positive integers and let $P=P_{s c+1}^{s d+1}, Q=Q_{r c+1}^{r d}$ be two homogeneous poly- 
nomials of $R\langle x, y\rangle$, of degrees $(s c+1)$ and $(r c+1)$ respectively and constant $x$ degrees $(s d+1)$ and $r d$ respectively, so that $[P, Q]=P Q-Q P=0$. Then either $P=0$ or $Q=0$.

Proof. Let us assume that both $P$ and $Q$ are not zero. As they are homogeneous polynomials which commute with each other, by Lemma 1 , there is a nonzero homogeneous polynomial $K$ of degree $k$ and nonzero constants $\alpha, \beta, \lambda, \mu$ so that $\alpha P=\lambda K^{m}, \beta Q=\mu K^{n}, m, n$ are nonnegative integers. As $P$ and $Q$ have constant $x$-degrees, $K$ also has constant $x$-degree, say $d_{x}(K)=h$, with $h \leqq k=d(K)$, then we have

$$
\alpha P_{s c+1}^{(s d+1)}=\lambda\left(K_{k}^{(h)}\right)^{m}, \quad \beta Q_{r c+1}^{(r d)}=\mu\left(K_{k}^{(h)}\right)^{n} .
$$

Notice that as $(s d+1) \geqq 1$ it is necessary that $h>0$. From $\left(^{*}\right)$ we get the set of equations

$$
\begin{aligned}
1 & =h m-s d, \\
1 & =k m-s c, \\
h n & =r d, \\
1 & =k n-r c .
\end{aligned}
$$

From (1) and (4) it follows that $d$ is relatively prime to $h$ and that $r$ is relatively prime to $n$; so, using (3) we get

$$
d=n, \quad h=r .
$$

If we multiply (1) by $k$ and (2) by $h$ we obtain

$$
\begin{aligned}
& k=k h m-k s d, \\
& h=h k m-s h c,
\end{aligned}
$$

and subtracting $\left(1^{\prime}\right)$ from $\left(2^{\prime}\right)$ we get $s(k d-h c)=h-k$, which, using (5), becomes $s(k n-r c)=h-k$. As, by (4), $k n-r c=1$, we finally obtain $s=h-k \leqq 0$ which is a contradiction since $s$ is a positive integer. That contradiction arises from the assumption that both $P$ and $Q$ are nonzero so either $Q$ or $P$ is equal to zero. This completes the proof.

TheOREM. Let $x+P$ and $y+Q$ be two polynomials of $R\langle x, y\rangle$. Let $P=P_{2}+P_{3}$ $+\cdots+P_{m}, Q=Q_{2}+Q_{3}+\cdots+Q_{n}$ be the decompositions of $P$ and $Q$ respectively into its homogeneous components, $m \geqq 2, n \geqq 2$. Let

$$
P_{r}=P_{r}^{(0)}+P_{r}^{(1)}+\cdots+P_{r}^{(r)}, \quad Q_{s}=Q_{s}^{(0)}+Q_{s}^{(1)}+\cdots+Q_{s}^{(s)},
$$

be the decompositions of $P_{r}$ and $Q_{s}$ respectively into its components of constant $x$-degree. Let us assume

(i) $P^{(0)}=Q^{(0)}=0$,

(ii) $[x+P, y+Q]=[x, y]$.

Then $P=0$ and $Q=f(x)$. 
Proof. We want to prove that $P_{r}^{(k)}=0$ for all $0 \leqq k \leqq r, r \geqq 2$ and that $Q_{r}^{(h)}=0$ for all $0 \leqq h \leqq r-1, r \geqq 2$.

1. The relation (ii) gives a set of equations, between them:

$$
\begin{aligned}
& \mathscr{E}_{r}^{s}:\left[P_{r-1}^{(s)}, y\right]+\sum_{k=2}^{r-2} \sum_{n=1}^{\min (k, s-1\}}\left[P_{r-k}^{(s-h)}, Q_{k}^{(h)}\right]+\left[x, Q_{r-1}^{(s-1)}\right]=0 \\
& \text { for } r \geqq 3,2 \leqq s \leqq r-1 .
\end{aligned}
$$

(Notice that as $P^{(0)}=Q^{(0)}=0$ we suppress the cases $h=0$ and $h=s$.)

2. $\mathscr{E}_{r}^{1}$ gives only $\left[P_{r-1}^{(1)}, y\right]=0, r \geqq 3$. The corollary to Lemma 1 implies that $P_{r-1}^{(1)}$ is a function of $y$ alone and since $r-1 \geqq 2$ this is not the case and so $P_{r-1}^{(1)}=0$. As a result $P^{(1)}=0$.

Consequently if $M=\max \{d(P), d(Q)\}, 1$ and 2 have reduced the problem to the proof of the following assertion:

(iii) $P_{m}^{n}=Q_{m}^{n-1}=0$ for all $M \geqq m \geqq n \geqq 2$.

3. For each pair of polynomials, $\left(P_{a}^{b}, Q_{a}^{b-1}\right), M \geqq a \geqq b \geqq 2$, define $\chi\left(P_{a}^{b}, Q_{a}^{b-1}\right)$ to be the rational number $(b-1) /(a-1)$.

Claim 1. Let $(b-1)$ and $(a-1)$ be two relatively prime positive integers. Then $\chi\left(P_{m}^{n}, Q_{m}^{n-1}\right)=(b-1) /(a-1), M \geqq m \geqq n \geqq 2$, if and only if there is a positive integer $r$ so that $m=r(a-1)+1, n=r(b-1)+1$.

The proof of Claim 1 is straightforward.

Observe that $S=\left\{\chi\left(P_{m}^{n}, Q_{m}^{n-1}\right), M \geqq m \geqq n \geqq 2\right\}$ is a finite set of rational numbers and as such there is an inherited natural ordering between its elements, namely that of the rationals. As a consequence we can prove (iii) by induction using the ordering of $S$.

4. Notice that $\chi\left(P_{M}^{2}, Q_{M}^{1}\right)<\chi\left(P_{m}^{n}, Q_{m}^{n-1}\right)$ for all $M \geqq m \geqq n \geqq 2$, unless $m=M$ and $n=2$. Consequently as the first step of our induction we have to prove that $P_{M}^{2}=Q_{M}^{1}$ $=0$. We have

$$
\mathscr{E}_{2 M}^{3}:\left[P_{M}^{(2)}, Q_{M}^{(1)}\right]=0, \quad \mathscr{E}_{M+1}^{2}:\left[P_{M}^{(2)}, y\right]+\left[x, Q_{M}^{(1)}\right]=0
$$

Using Lemma 3 for the case $c=M-1, d=s=r=1$, from $\mathscr{E}_{2 M}^{3}$ we get that either $P_{M}^{(2)}$ or $Q_{M}^{(1)}$ is equal to zero, and from $\mathscr{E}_{M+1}^{2}$ and the corollary to Lemma 1 it follows that both $P_{M}^{(2)}$ and $Q_{M}^{(1)}$ are zero.

5 . Let us assume now that given $(a-1)$ relatively prime to $(b-1), M \geqq a \geqq b \geqq 2$, we have proved that (iii) holds whenever $\chi\left(P_{m}^{n}, Q_{m}^{n-1}\right)<(b-1) /(a-1), M \geqq m \geqq n \geqq 2$. We want to prove now (iii) for $\chi\left(P_{m}^{n}, Q_{m}^{n-1}\right)=(b-1) /(a-1)$. In view of Claim 1 we only have to prove that $P_{r(a-1)+1}^{r(b-1)+1}=Q_{r(a-1)+1}^{r(b-1)}=0$ for any positive integer $r$. For this purpose consider the set of equations $\mathscr{E}_{r(a-1)+2}^{r(b-1)+1}$ for all positive integers $r$.

A typical summand in $\mathscr{E}_{r(a-1)+2}^{r(b-1)+1}$ is

$$
\left[P_{r(a-1)+2-k}^{r(b-1)+1-h}, Q_{k}^{h}\right] ; \quad 2 \leqq k \leqq r(a-1), 1 \leqq h \leqq k, h \leqq r(b-1) .
$$


Claim 2. Either

(1) $(r(b-1)-h) /(r(a-1)+1-k) \leqq(b-1) /(a-1)$ or

(2) $h /(k-1) \leqq(b-1) /(a-1)$

and in (1) equality holds iff equality holds also in (2).

Proof of Claim 2. If (1) does not hold it means that $(r(b-1)-h) /(r(a-1)+1-k)$ $>(b-1) /(a-1)$ or else

(3) $(r(b-1)-h)(a-1)>(b-1)(r(a-1)+1-k)$

which, after simplifying, becomes

(4) $h(a-1)<(b-1)(k-1)$

which is simply (2).

In the event of having equality in (1) it is clear that (3) and so (4) will also be equalities, which completes the proof of Claim 2.

It follows then that in case (1) is a strict inequality we have $P_{r(a-1)+2-h}^{r(b-1)+1-h}=0$ by inductive hypothesis; and in case (1) does not hold then (2) is a strict inequality and so, again by inductive hypothesis, we obtain that $Q_{k}^{h}=0$. Finally there remain the cases in which (1) and hence (2) are equalities, but that implies that $\mathscr{E}_{r(a-1)+2}^{r(b-1)+1}$ is a sum of terms which have the same $\chi=(b-1) /(a-1)$, i.e. $\mathscr{E}_{r(a-1)+2}^{r(b-1)+1}$ becomes

$$
\left[P_{r(a-1)+1}^{r(b-1)+1}, y\right]+\sum_{n=1}^{r-1}\left[P_{(r-n)(a-1)+1}^{(r-n)(b-1)+1}, Q_{n(a-1)+1}^{n(b-1)}\right]+\left[x, Q_{r(a-1)+1}^{r(b-1)}\right]=0 .
$$

We want to have

$$
(k): P_{k(a-1)+1}^{k(b-1)+1}=Q_{k(a-1)+1}^{k(b-1)}=0 \text { for all positive integers } k .
$$

As we have a finite set of polynomials, there exists $N$ so that $(k)$ holds whenever $k>N$; if we can prove that $(N)$ holds, then by a repeated argument we will have that $(k)$ holds for all positive integers $k$.

$\mathscr{E}_{2 N(a-1)+2}^{2 N(b-1)+1}$ reduces to the single summand $\left[P_{N(a-1)+1}^{N(b-1)+1}, Q_{N(a-1)+1}^{N(b-1)}\right]=0$ which, by Lemma 3, for $c=a-1, d=b-1, r=s=N$, implies that either of the factors is zero. Assume first that $Q_{N(a-1)+1}^{N(b-1)}=0$ and that $P_{N(a-1)+1}^{N(b-1)+1} \neq 0$. We can prove that in this case

(iv) $Q_{(N-h)(a-1)+1}^{(N-h)(b-1)}=0$ for all $0 \leqq h \leqq N-1$.

In fact for $h=0$ we had assumed (iv) to be true. Let us suppose that (iv) holds for all $0 \leqq h<h_{0} \leqq N-1$ and let us obtain that it must also hold for $h=h_{0}$. The equation $\mathscr{E}_{\left[N+\left(N-h_{0}\right)\right](a-1)+2}^{\left.\left[N+N-h_{0}\right)\right](b-1)+1}$ reduces to the single term $\left[P_{N(a-1)+1}^{N(b-1)+1}, Q_{\left(N-h_{0}\right)(a-1)+1}^{\left(N-h_{0}\right)(b-1)}\right]=0$ and, as $P_{N(a-1)+1}^{N(b-1)+1} \neq 0$, it follows from Lemma 3, for $c=a-1, d=b-1, s=N, r=N-h_{0}$, that $Q_{\left(N-h_{0}\right)(b-1)}^{(N-1)+1}=0$ which is (iv) $h=h_{0}$.

As we have obtained that (iv) holds for all $0 \leqq h \leqq N-1$, equation $\mathscr{E}_{N(a-1)+2}^{N(b-1)+1}$ reduces to the single summand $\left[P_{N(a-1)+1}^{N(b-1)+1}, y\right]=0$, and from here it is clear that $P_{N(a-1)+1}^{N(b-1)+1}$ must also be zero (corollary to Lemma 1 ).

Using a completely similar argument we can discard the possibility that $Q_{N(a-1)+1}^{N(b-1)} \neq 0$ and $P_{N(a-1)+1}^{N(b-1)+1}=0$ and consequently the only possibility that remains 
is that both of them are zero. This proves that $P_{r(a-1)+1}^{r(b-1)+1}=Q_{r(a-1)+1}^{r(b-1)}=0$ for all positive integers $r$, which completes the induction and hence the proof of the theorem.

COROLlaRY. Let $\varphi$ be an automorphism of $R\langle x, y\rangle$ so that $\varphi(x y-y x)=\lambda(x y-y x)$, $\lambda$ a unit of $R$. Then $\varphi$ is tame.

Proof. Notice first that any elementary automorphism of $R\langle x, y\rangle$ keeps $(x y-y x)$ fixed (modulo a unit of $R$ ), so without loss of generality we can assume $\varphi(x)$ $=x+P(x, y), \varphi(y)=y+Q(x, y)$, where $P$ and $Q$ do not contain terms of zero nor of first degree (see Lemma 2 and the following remarks).

Let $R(\tilde{x}, \tilde{y})$ be the free commutative and associative algebra over $R$, with generators $\tilde{x}$ and $\tilde{y}$. Let $\sigma: R\langle x, y\rangle \rightarrow R(\tilde{x}, \tilde{y})$ be the unique $R$-homomorphism which sends $x$ into $\tilde{x}$ and $y$ into $\tilde{y}$. Define $\tilde{\varphi} \in \operatorname{End}_{R}(R(\tilde{x}, \tilde{y}))$ as follows:

$$
\tilde{\varphi}(x)=\sigma(\varphi(x))=\tilde{x}+\tilde{P}(\tilde{x}, \tilde{y}), \quad \tilde{\varphi}(y)=\tilde{y}+\tilde{Q}(\tilde{x}, \tilde{y}) .
$$

It is clear that if $\varphi$ is an automorphism of $R\langle x, y\rangle$ then $\tilde{\varphi}$ is an automorphism of $R(\tilde{x}, \tilde{y})$. So we may now apply Jung's Theorem [6] which says that every $R$ automorphism of $R(\tilde{x}, \tilde{y})$ is a product of elementary automorphisms of $R(\tilde{x}, \tilde{y})$ and we obtain that $\tilde{\varphi}$ is a product of elementary automorphisms of $R(\tilde{x}, \tilde{y})$. Observe that if $\pi$ is an elementary automorphism of $R(\tilde{x}, \tilde{y})$ there exists a well-defined elementary automorphism of $R\langle x, y\rangle$, say $\pi^{*}$, so that $\left(\pi^{*}\right)^{\sim}=\pi$. Let $\varphi^{*}$ be a tame automorphism of $R\langle x, y\rangle$ so that $\left(\varphi^{*}\right)^{\sim}=\tilde{\varphi}$. Then $\varphi=\tau \circ \varphi^{*}$ where $\tau$ is an automorphism of $R\langle x, y\rangle$ so that

(1) $\tau=$ identity of $R(\tilde{x}, \tilde{y})$.

In particular we may write $\tau(x)=x+M(x, y), \tau(y)=y+N(x, y)$, where $M$ and $N$ are free of terms of the zero and the first degrees. It is also clear that $\tau(x y-y x)$ $=x y-y x$ and from (1) it follows that $M^{(0)}=N^{(0)}=0$. Then by the theorem we obtain the following equalities:

$$
\tau(x)=x, \quad \tau(y)=y+N(x) .
$$

But (1) implies that $N(x)=0$ and hence $\tau=$ identity of $R\langle x, y\rangle$. Consequently $\varphi=\varphi^{*}$ which is tame. This completes the proof.

\section{BIBLIOGRAPHY}

1. P. M. Cohn, Free associative algebras, Bull. London Math. Soc. 1 (1969), 1-39. MR 39 \#2800.

2. - On subsemigroups of free semigroups, Proc. Amer. Math. Soc. 13 (1962), 347-351.

3. - Subalgebras of free associative algebras, Proc. London Math. Soc. (3) 14 (1964), 618-632. MR 29 \#4777.

4. - - Lecture notes on free rings, Yale University, New Haven, Conn., 1962. 
5. G. M. Bergman, Centralizers in free associative algebras, Trans. Amer. Math. Soc. 137 (1969), 327-344. MR 38 \#4506.

6. H. W. E. Jung, Über ganze birationale Transformationen der Ebene, J. Reine Angew. Math. 184 (1942), 161-174. MR 5, 74.

7. W. Magnus, A. Karrass and D. Solitar, Combinatorial group theory: Presentations of groups in terms of generators and relations, Pure and Appl. Math., vol. 13, Interscience, New York, 1966. MR 34 \#7617.

Courant Institute of Mathematical Sciences, New York University, NeW YORK, NEW YORK 10012 